\title{
OFDM PAPR Reduction Using Recurring SLM with Cyclic and Linear Block Codes Schemes
}

\author{
Pratima Manhas**1, M. K Soni ${ }^{2}$ \\ FET, ECE Deptt, Manav Rachna International University, Faridabad, India

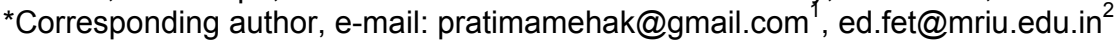

\begin{abstract}
Orthogonal Frequency division multiplexing is a type of multicarrier modulation technique which is used in various wireless \& telecommunication standards. In OFDM, higher data rate is split into several narrowband data stream that is transmitted simultaneously over a number of subcarriers. One major disadvantage of using OFDM is high Peak to Average power ratio. This paper presents the simulation result which compares the PAPR using SLM with Cyclic \& Linear block codes. The simulation study verifies that the PAPR reduction by proposed method is better than the existing technique (SLM).
\end{abstract}

Keywords: orthogonal frequency division multiplexing (OFDM), selective mapping (SLM), peak-toaverage-power-ratio (PAPR), linear block codes, cyclic code.

Copyright $\odot 2015$ Institute of Advanced Engineering and Science. All rights reserved.

\section{Introduction}

Orthogonal Frequency Division Multiplexing (OFDM) is a multicarrier modulation method which is widely used in wireless communication. It is used in mobile communication, Digital terrestrial mobile communication, Digital Audio Broadcasting (DAB), Digital Video Broadcasting terrestrial (DVB-T), wireless asynchronous transfer mode (WATM), Modem/ADSL. The transmit signals in an orthogonal frequency-division multiplexing (OFDM) system can have high peak values in the time domain since many subcarrier components are added via an inverse fast Fourier transformation (IFFT) operation [1]. As a result, OFDM systems are known to have a high peak-to-average power ratio (PAPR) when compared to single-carrier systems. High value of PAPR will cause clipping in OFDM signal and enable an amplifier to operate in nonlinear section. Due to presence of this nonlinearity it will result in in-band distortion and outband radiation. The in -band distortion will lead to degrade the performance of the system and out-band radiation result in causing interference among adjacent channel. Thus this additional interference in the system will results in increasing Bit Error Rate. One way to overcome this situation is to use high dynamic range linear power amplifier, but utilization of this solution is not effective, and too expensive.

\subsection{Orthogonal Frequency Division Multiplexing Model}

OFDM can be viewed as both modulation \& multiplexing technique.Modulation can be viewed as a relation between input \& output while the multiplexing can be viewed as the output which is a linear sum of input. OFDM differs from FDM. In FDM, entire bandwidth is used by user, while in OFDM bandwidth is divided into many narrow band channels and each is allocated to user. Hence OFDM supports more subscribers/channels compare to FDM. In FDM systems the carriers are far apart with respect to each other and in OFDM systems carriers are densely packed and are orthogonal to the other carriers. Orthogonal means peak of one carrier occurs at null of the other. Hence OFDM system is bandwidth efficient as compared to the FDM system. The OFDM model for both transmitter \& receiver is shown in Figure 1 and 2. 


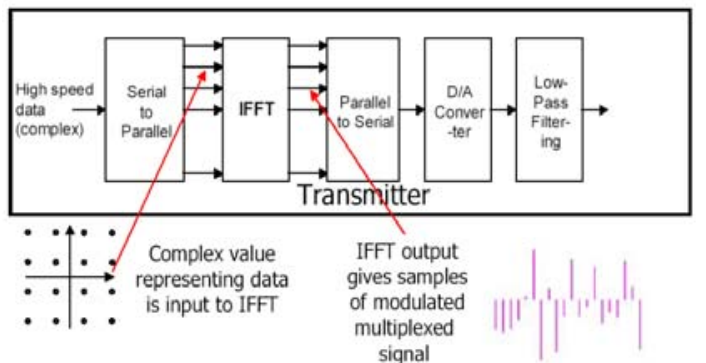

Figure 1. OFDM Transmitter

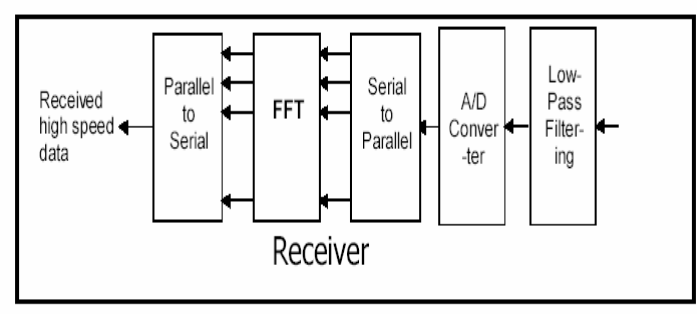

Figure 2. OFDM Receiver

The data is first converted into serial to parallel form and then each subcarrier is modulated by Quadrature Phase shift modulator (QPSK) modulation and then IFFT is performed. The received signal fed to serial to parallel converter and FFT is performed to get the original data signal. The OFDM system has various advantages such as have more resistant to selective fading, ability to recover from the interference and inter symbol and inter frame interference (ISI), effective and suitable utilization of available spectrum, channel equalization is easier [2,3]. The major drawback in OFDM method is high peak to average power ratio (PAPR), commonly the PAPR value for some transmitted signal is much larger than the usual values. This PAPR factor enables the amplifier to work in nonlinear section which results in degrading the performance of OFDM systems.

\subsection{PAPR of OFDM System}

The OFDM signal consists of multiple modulated carriers and able to generate a high peak to average power ration (PAPR), when these coherent carriers are added up $[4,5]$. If $\mathrm{N}$ carrier signals which are added together constitute same phase then they generate a peak power which is $\mathrm{N}$ times the average power. Thus, this increased value of PAPR will in turn responsible for clipping noise, non-linear distortion of power amplifiers, BER performance $[6,7]$ debasement, energy spilling into adjacent channels, inter modulation effects on the sub carriers, warping of signal constellation in each and every sub channel, enhanced the complexity factor in analog to digital or digital to analog convertor .The PAPR is defined as the ratio of Peak power to the average power. Equation (1) represents the PAPR of transmitted signal.

$$
P A P R=\frac{\max _{0 \leq t \leq T}|x(t)|^{2}}{1 / T \cdot \int_{0}^{T}|x(t)|^{2} d t}
$$

The cumulative distribution function (CDF) of the PAPR is one of the most frequently used performance measures for PAPR reduction techniques. The CCDF of the PAPR denotes the probability that the PAPR of a data block exceeds a given threshold.

The paper is organized in different sections. Section 2 represents the introduction of Cyclic \& Linear Block codes. A brief introduction to SLM technique \& modified SLM is presented in section 2. The comparison of both proposed method (cyclic \& linear block codes) and existing method is presented in section 3 . Lastly results are concluded in section 4 .

\section{Proposed Method}

A linear Block code is a code in which exclusive OR of the two valid code words creates another codeword. It is represented as in Equation (2).

$$
C=U . G
$$

Where, $\mathrm{U}=$ uncoded information word with $\mathrm{K}$ bits

$\mathrm{C}=$ Corresponding codeword for the information bits)

$\mathrm{G}=$ Generator matrix of $\mathrm{n} \times \mathrm{k}$ where $\mathrm{n}$ is the Column (output bits) \& $\mathrm{k}$ is the row (input

TELKOMNIKA Vol. 16, No. 1, October $2015: 1-6$ 
Let us take an example if the generator matrix is $\mathrm{G}=$ Here $\mathrm{n} \times \mathrm{k}=(7 \times 3)$

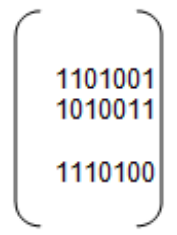

$C=\left(u 0\right.$ u1 u2). $\left(\begin{array}{c}1101001 \\ 1010011 \\ 1110100\end{array}\right)$

$C=u 0 .(1101001)+u 1 .(1010011)+u 2 .(1110100)$

In Linear block codes the addition used is modulo2 or the exclusive OR addition. Suppose we want to find the code words from $\mathrm{c} 0 \mathrm{t0} \mathrm{c} 7$, then is given as:

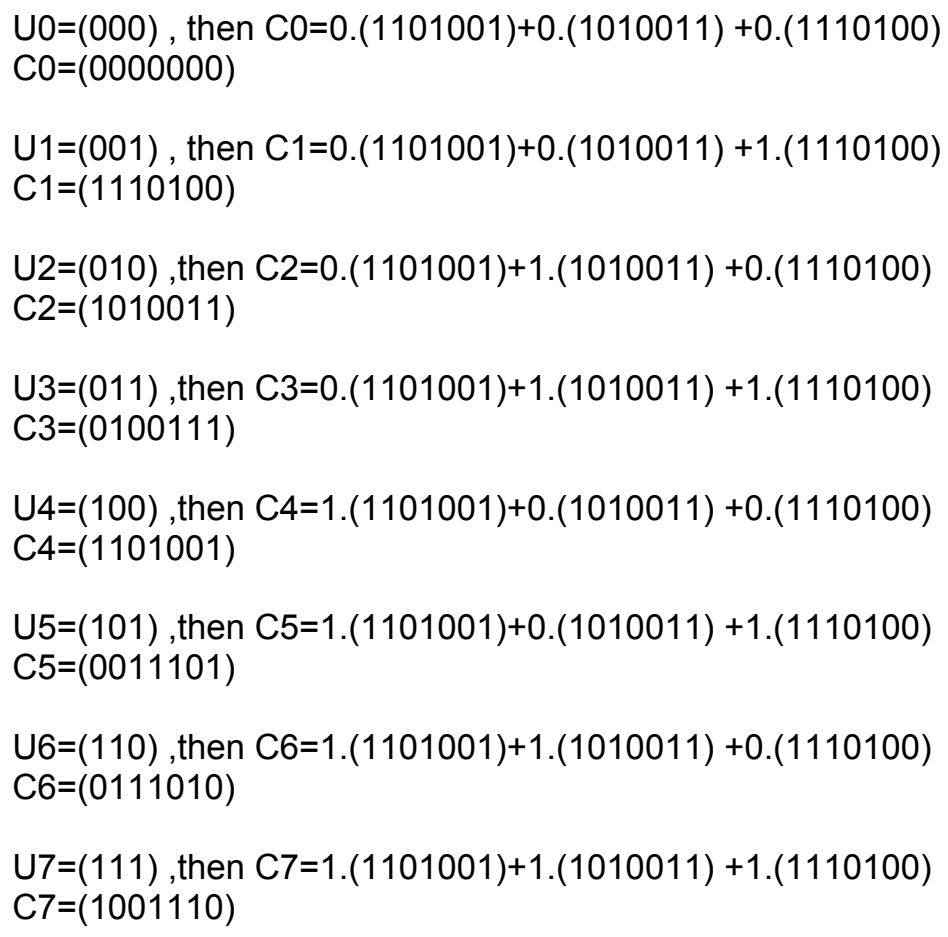

\subsection{Cyclic Codes}

Cyclic codes are the special type of linear block codes with one extra property. In cyclic code, if a codeword is cyclically shifted (rotated), the result is another codeword [8]. It can be implemented using simple shift registers. The cyclic shift is given as:

Suppose $x=10110$

$\Pi(x)=01011$

Here in this example the last bit is shifted to the first.

A cyclic code has the following advantages:

1) It has better minimum distance than other linear codes with same parameters

2) Encoding \& decoding is performed by simple feedback circuitry.

3) It can be easily implemented and has cost effective electronic circuit. 


\subsection{Proposed SLM Technique with Cyclic/Linear Block Codes}

In SLM method the transmitter selects one favorable transmit signal from a set of sufficiently different signals which all represent the same information[9][10]. The systematic diagram for SLM method and modified SLM is shown in Figure $3 \& 4$. The data stream after serial to parallel conversion is represented by vector $X=\left[X_{0}, X_{1}-------, X_{N-1}\right]^{\top}$. Then phase rotated data due to the phase rotation factor $\mathrm{B}^{(\mathrm{u})}$ can be written as:

$$
x^{(u)}=\operatorname{IFFT}\left(\mathrm{x}^{\prime} \mathrm{B}^{(\mathrm{u})}\right)
$$

Where,

$$
B^{(u)}=\left[B_{0}{ }^{(u)}, B_{1}{ }^{(u)},-------, B_{N-1}{ }^{(u)}\right)^{\top}
$$

$\mathrm{u}=0,1,---\mathrm{U}-1$ is the phase weighting sequence with $\left|\mathrm{B}_{\mathrm{n}}{ }^{(\mathrm{u})}\right|=1(\mathrm{n}=0,1, \mathrm{~N}-1)$ and is usually selected from $\{ \pm 1\}$ for avoiding the complexity for complex multiplication. The modified data for the $\mathrm{u}^{\text {th }}$ phase sequence is given as:

$$
X(u)=\left[X_{0} B^{u}{ }_{0}, X_{1} B^{u}{ }_{, 1},-----, X_{N-1} B^{u}{ }_{N-1}\right]^{\top}
$$

After that the PAPR comparison among the $U$ data sequence $x^{(u)}$ is done and the optimal mapped one $\hat{X}$ with the minimum PAPR is selected for transmission.

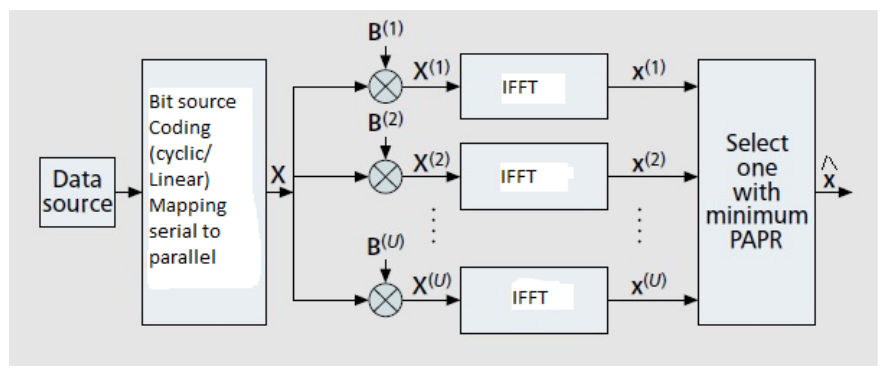

Figure 3. Block diagram of SLM technique

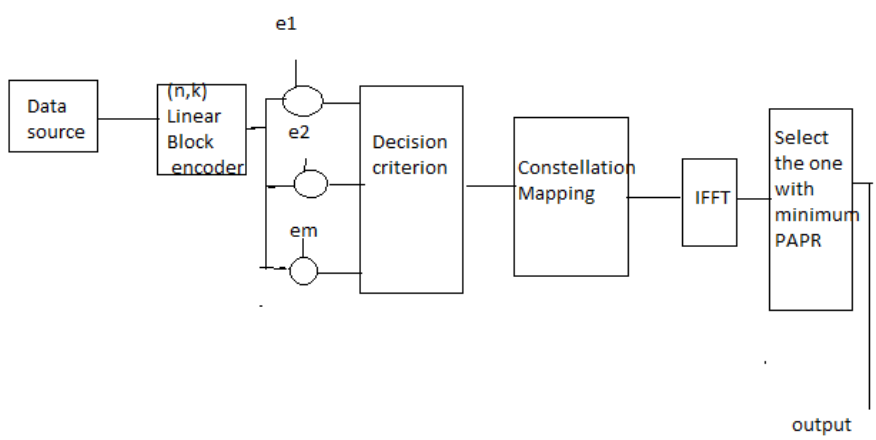

Figure 4: Block diagram of SLM using Linear Block codes

\section{Proposed Work}

The mapping sequence for both cyclic \&linear block codes are selected to decrease the peak to average power ratio (PAPR) of OFDM signal. SLM method have higher complexity factor as it includes large number of IFFT operations. MATLAB tool is used to calculate CCDF and Power spectral density (PSD) for both existing SLM and proposed SLM(using cyclic \& Linear block codes) [12]. Table 1 shows the simulation parameters. 
Table1. Simulation Parameters

\begin{tabular}{ll}
\hline Modulation & QPSK \\
\hline Number of data subcarriers(N) & 256 \\
Total number of data symbols & 1000 \\
Size of the phase sequence & 256 \\
FFT Size & 64 \\
Coding Technique & Cyclic\& Linear \\
\hline
\end{tabular}

\section{Results \& Analysis}

Figure 5 and 7 represents the CCDF plot of PAPR for existing SLM (QPSK based OFDM) and modified SLM (both cyclic \& Linear codes). Figure 6 and 8 represents the comparison between Power spectral density of existing SLM and Proposed SLM (both cyclic \& Linear codes). Then the result is compared with the existing SLM technique and found that the proposed work has effective outcome than the existing SLM method.

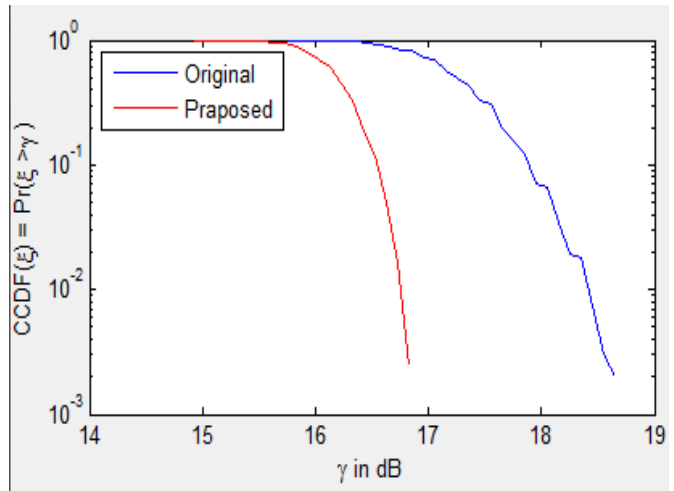

Figure 5. CCDF plot of PAPR of basic QPSK-OFDM system with the SLM technique and Proposed Work (with cyclic)

By using this phase sequence [1, $-1, \mathrm{j},-\mathrm{j}]$ obtained PAPR by using proposed SLM method is nearly about $17 \mathrm{~dB}$ (Figure 5) and $16 \mathrm{~dB}$ (Figure 7). On other hand the PAPR obtained by existing SLM method is nearly 19dB (in Figure 5 and 7). Thus, by proposed SLM method enables to reduce the PAPR by nearly $2 \mathrm{db}$ and $3 \mathrm{~dB}$ which is $11.76 \%$ \& $18.75 \%$ in percentage improvement over original method respectively.

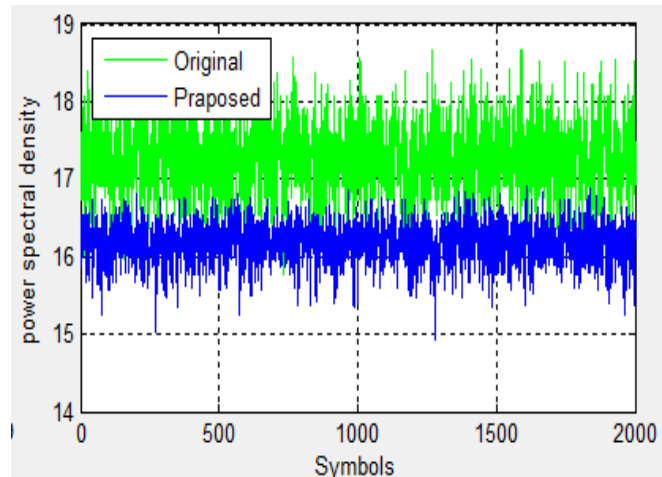

Figure 6. Comparison of PSD between original QPSK-OFDM PAPR with SLM \& PAPR with Proposed Work (with cyclic)

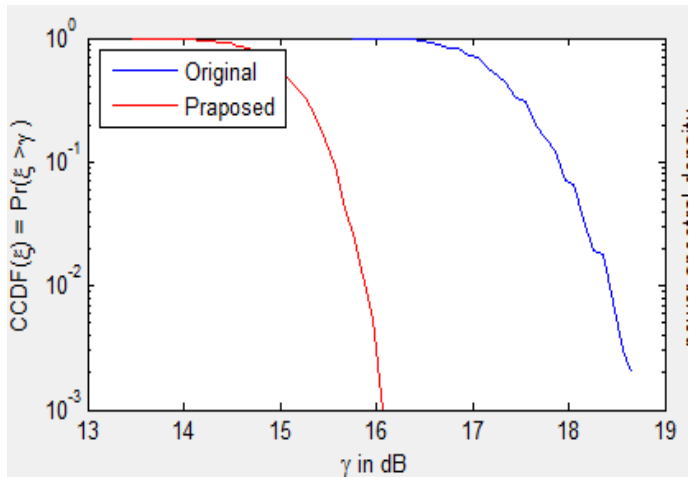

Figure 7. CCDF plot of PAPR of basic QPSKOFDM system with the SLM technique and Proposed Work (with linear code) 


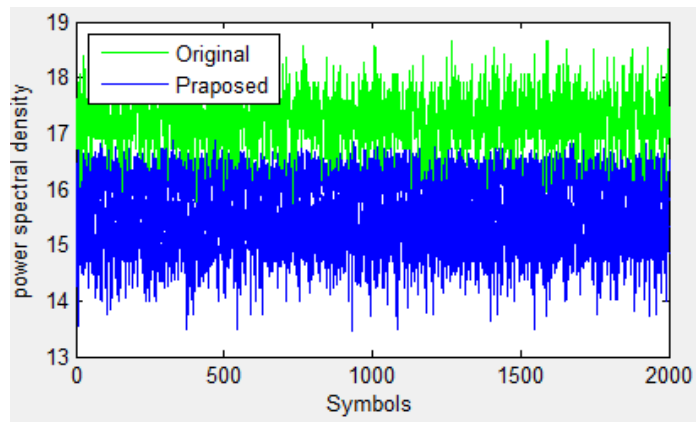

Figure 8. Comparison of PSD between original QPSK-OFDM PAPR with SLM \& PAPR with Proposed Work (with linear code)

\section{Conclusion}

The modified SLM method by using both cyclic \& linear block codes which has better performance in terms of CCDF and PSD with respect to existing SLM is shown in this paper. Figure 5 and 7 represents the results obtained by the proposed method for reducing PAPR. The comparison between the modified SLM using cyclic code results in $2 \mathrm{db}$ reduction in PAPR while in case of modified SLM using Linear block code reduces the PAPR by $3 \mathrm{db}$. MATLAB tool is used to obtain the performance of PAPR reduction, which clearly represents that there is a significant improvement in performance. The proposed methods in this paper will results in reduction of PAPR by $2 \mathrm{~dB} \& 3 \mathrm{db}$ which is an improvement of $11.76 \% \& 18.75 \%$ in all.

\section{References}

[1] Wu, WY Zou. Orthogonal frequency division multiplexing: A multi-carrier modulation scheme. IEEE Trans. Consumer Electronics. 1995; 41(3): 392-399.

[2] Nee, Richard van, Ramjee Prasad. OFDM for wireless multimedia communications. Artech House, Inc. 2000.

[3] Debbah, Mérouane. Short introduction to OFDM. White Paper, Mobile Communications Group, Institut Eurecom. 2004.

[4] Filbert Juwono, Randy S Putra, Dadang Gunawan. A Study on PeaktoAverage Power Ratio in DWTOFDM Systems. TELKOMNIKA Indonesian Journal of Electrical Engineering. 2014; 12: 39553961.

[5] Muhmmad Anjum, Mussa A Dida, MA Shaheen. Peak Power Reduction Using Improved Selective Mapping Technique for OFDM. TELKOMNIKA Indonesian Journal of Electrical Engineering. 2014; 12: 6291-6296.

[6] Heo Seok-Joong, et al. A modified SLM scheme with low complexity for PAPR reduction of OFDM systems. Personal, Indoor and Mobile Radio Communications, 2007. PIMRC 2007. IEEE 18th International Symposium on IEEE. 2007.

[7] Chen Houshou, Hsinying Liang. Combined selective mapping and binary cyclic codes for PAPR reduction in OFDM systems. Wireless Communications, IEEE Transactions. 2007: 3524-3528.

[8] Shinsuke Hara, Ramjee. Principle and history of MCM/OFDM. In Multicarrier techniques for 4G mobile communication, Artech House.

[9] H Sampath, et al. A fouth-generation MIMO-OFDM broadband wireless system: design, performance and field trial results. IEEE Communication Magazine. 2002; 40(9).

[10] D Wulich. Reduction of peak to mean ratio of multicarrier modulation using cyclic coding. Electronic Letters. 1996; 32: 432-433.

[11] Wulich Dov. Definition of efficient PAPR in OFDM. Communications Letters, IEEE. 2005; 9(9): 832834.

[12] Yang, Kyeongcheol, Seok-II Chang. Peak-to-average power control in OFDM using standard arrays of linear block codes. Communications Letters, IEEE. 2003; 7(4): 174-176.

[13] Amit Shukla, Vineeta Saxena Nigam. PAPR Reduction in OFDM System Based on SLM Technique. International Journal of Innovative Technology and Exploring Engineering (IJITEE). 2013; 3(4).

[14] Dae-Woon Lim, Jong-Seon No, Chi-Woo Lim, Habong Chung. A New SLM OFDM Scheme with Low Complexity for PAPR Reduction. IEEE Signal Processing Letters. 2005; 12(2).

[15] Shao Kai, Guo Zhigang, Zhuang Ling, Wang Guangyu. An Effective Scheme for PAPR Reduction of OFDM Signal Using Time-varying Subcarrier. IEEE. 2013

[16] Leonard J Cimini Jr, Nelson R Sollenberger. Peak-to-Average Power Ratio Reduction of an OFDM signal using partial transmit sequences with embedded side information. Global Telecommunications Conference. 2000; 2: 746-750.

TELKOMNIKA Vol. 16, No. 1, October 2015: $1-6$ 